Patients will be required to meet specific criteria for initiation of FGM as per the National Institute of Clinical Excellence (NICE- NG 18) guidelines. Patients would also be required to meet specific measures of improvement in diabetic control for continuation of FGM under the National Health Service (NHS) funded services. These include completing regular scanning of sensor, sensor use time of more than $70 \%$, improvement in baseline $\mathrm{HbA} 1 \mathrm{c}$ and reduction in diabetes related hospital attendances or admissions.

Objectives To review the compliance and effectiveness of FGM use amongst paediatric patients with T1DM, within our service.

Methods This was a retrospective audit. A total of 46 patients were commenced on FGM between April 2019 and April 2020.

We collected and analysed the data, focusing on the percentage time of sensor use, number of scans per day, HbA1c at $0,1,3,6,9$ and 12 months and the average number of diabetes related hospital attendances or admissions per year before and after starting FGM.

Results Forty-six patients were started on FGM due to poor glucose monitoring, persistently high $\mathrm{HbA1c}$ and frequent hypoglycaemic episodes. The mean age of these children at the start of FGM was 12.8 years.

Of the total 46 patients, 28 (61\%) continued using FGM while the remaining 18 discontinued FGM. The reasons for discontinuing included primary skin conditions affecting adhesiveness of device (2 patients), changing to continuous glucose monitoring system (6 patients), nonengagement in the technology ( 2 patients) and personal choice (8 patients).

Out of these 28 patients, $18(64.3 \%)$ patients had sensor usage time of more than $70 \%$ and 8 (28.6\%) did more than 8 scans per day. On commencing FGM (0 months), the HbA1c of these 28 children ranged between 6.2 and $11.2 \mathrm{mmol} / \mathrm{mol}($ mean $=8.8)$. At 12 months, this was between 5.8 to $11.8 \mathrm{mmol} / \mathrm{mol}($ mean $=8.3)$, demonstrating a $0.5 \mathrm{mmol} / \mathrm{mol}$ reduction in the mean. A One-tailed Mann-Whitney U-test indicates that the HbA1c at 12 months is less than at 0 months, $U=279, p=0.07$. While this may not be statistically significant, we have demonstrated that after commencing FGM, 19 (67.9\%) patients' serial HbA1c displayed a downward trend, and 11 patients (39.2\%) had a reduction in diabetes related hospital attendances per year, with both criteria being met by 8 (28.5\%) patients.

Conclusions Our results demonstrate that FGM helps improve diabetes management and outcomes. The treating diabetes teams should continue to support patients in the use of advanced technology through regular follow-up, and empower patients and carers towards diabetes self-management, thereby improving outcomes.

\section{CASE SERIES OF CHILDREN PRESENTING WITH ACUTE VISUAL IMPAIRMENT}

Hnin Wint Wint Aung, Kyaw Linn. Myanmar

\subsection{6/bmjpo-2021-RCPCH.197}

Background Acute or rapidly progressive visual impairment in children is a medical emergency which needs early recognition and optimal management strategies. Among the different causes of acute visual impairment in children, optic neuritis $(\mathrm{ON})$ is an acquired disorder of the optic nerve due to inflammation, demyelination or degeneration and it is treatable. It may also represent a sentinel attack or relapse of a chronic demyelinating disorder such as multiple sclerosis (MS), neuromyelitis optica spectrum disorder (NMOSD) and myelin oligodendrocyte glycoprotein (MOG) antibody associated diseases.

Objectives This is to report a case series of three children presenting with acute visual impairment due to optic neuritis.

Methods This is a retrospective, descriptive case series by reviewing medical records of initial admission and follow-up.

Results Three patients were included in this case series.

Case 1: a 6-year-old girl suffered from acute visual impairment which was started on the right side and then involved bilaterally. Other systemic and neurological examinations were normal. Visual acuity was just perception of light on both eyes. She had a previous episode of acute disseminated encephalomyelitis (ADEM) 4 months ago. Investigations revealed positive MOG-IgG with negative anti-aquaporin 4 (AQP4) antibodies. MRI brain showed T2 hyperintense lesions with contrast enhancement of bilateral optic nerves. She was diagnosed as MOG-optic neuritis. Her vision fully recovered with intravenous methylprednisolone and immunoglobulin.

Case 2: a 10-year-old girl presented with a relapsing bilateral optic neuritis. At the first presentation, her visual acuity was perception to light only on both eyes. Anti-aquaporin 4 antibody was positive and bilateral optic nerves enlargement with contrast enhancement was seen in MRI brain. Her vision almost fully recovered with intravenous methylprednisolone. She had relapsed with acute visual impairment, intractable hiccups and vomiting, 3 months later. She was diagnosed as bilateral optic neuritis with area postrema syndrome; therefore, the second cycle of intravenous methylprednisolone was started again together with mycophenolate mofetil for longterm immunosuppression. However, her vision only partially recovered with visual acuity $6 / 20$ on the right side and $6 / 60$ on the left.

Case 3: a 7.5-year-old boy presented with acute onset of bilateral visual impairment with sensory impairment below T4 level. His blood inflammatory markers were normal and antiaquaporin 4 and anti-MOG antibodies were also negative. Features of bilateral optic neuritis and transverse myelitis in spinal cord from C4 to T5 were seen in MRI studies. The diagnosis was NMOSD with negative AQP4-IgG. His vision also fully recovered after receiving intravenous methylprednisolone therapy.

Conclusions Optic neuritis should always be considered in children with acute visual impairment. Complete neurological and ophthalmological examinations are essential features of the clinical evaluation. Ophthalmology referral is required. Magnetic resonance imaging (MRI) study of the brain and orbits with gadolinium contrast provides confirmation of the diagnosis and underlying causes in most cases. Other tests including lumbar puncture, antibody testing for MOG and AQP4 antibodies are also recommended. Accurate and early diagnosis of optic neuritis is crucial as prompt aggressive treatment may result in a good prognosis on vision and mitigation of long-term disability. 